\title{
Teor de Taninos Condensados Presente na Casca de Jurema-Preta (Mimosa tenuiflora) em Função das Fenofases
}

\author{
Tatiane Kelly Barbosa de Azevêdo ${ }^{1}$, Juarez Benigno Paes², Leandro Calegari ${ }^{3}$, \\ Gregório Mateus Santana ${ }^{4}$
}

${ }^{1}$ Departamento de Agropecuária, Universidade Federal do Rio Grande do Norte - UFRN, Macaíba/RN, Brasil ${ }^{2}$ Departamento de Ciências Florestais e da Madeira, Universidade Federal do Espírito Santo UFES, Jerônimo Monteiro/ES, Brasil

${ }^{3}$ Unidade Acadêmica de Engenharia Florestal, Universidade Federal de Campina Grande - UFCG, Patos/PB, Brasil ${ }^{4}$ Programa de Pós-graduação em Ciências Ambientais e Florestais, Universidade Federal Rural do Rio de Janeiro - UFRRJ, Seropédica/RJ, Brasil

\section{RESUMO}

A jurema-preta (Mimosa tenuiflora), espécie típica do semiárido brasileiro, possui potencial para a produção de taninos vegetais. Este estudo teve como objetivo avaliar o teor de taninos condensados na casca de jurema-preta em função das fenofases da planta. Assim, foram realizadas quatro coletas de acordo com o período agrícola, em agosto e dezembro de 2008 e em abril e agosto de 2009, na Fazenda Lameirão, Santa Terezinha, Paraíba, sendo coletadas 10 plantas por período, totalizando 40 plantas, das quais foram avaliadas a fenologia e nas quais foram realizadas as análises quanto ao teor de sólidos totais, índice de Stiasny e teor de taninos condensados na casca por período de coleta. Observou-se que a fenologia das plantas influenciou no teor de taninos condensados, pois quando as árvores tinham frutos e flores apresentaram menor teor de taninos. Já na presença de folhas verdes foi observado maior teor de taninos condensados nas plantas.

Palavras-chave: período agrícola, polifenóis, índice de Stiasny.

\section{Content of Condensed Tannins Present in Mimosa tenuiflora Barks Based on the Phenophase}

\begin{abstract}
The Mimosa tenuiflora, a typical species of the Brazilian semi-arid region, presents potential for production of vegetable tannins. This study aimed to evaluate the content of condensed tannins in the bark of Mimosa tenuiflora based on the plant phenophase. Thus, four collections were carried out according to the agricultural period, August and December of 2008, and April and August of 2009, at Lameirão Farm, Santa Terezinha, Paraiba, Brazil. Were collected 10 plants per period, totaling 40 plants, and evaluated the phenology and total solids content, Stiasny's index and condensed tannins content in the bark by collection period. It was observed that the plants phenology affected the content condensed tannins, since trees with fruits and flowers showed lower tannins content. Plants with green leaves showed higher condensed tannins content.
\end{abstract}

Keywords: agricultural period, polyphenols, Stiasny's index. 


\section{INTRODUÇÃO}

Os taninos vegetais, substâncias capazes de precipitar as proteínas presentes em peles e couros, podem ser encontrados em várias partes dos vegetais, como no cerne da madeira, na casca, nos frutos, nas folhas e nas sementes (Paes et al., 2010). São constituídos por polifenóis e classificados em hidrolisáveis e condensados. Os hidrolisáveis são poliésteres da glicose e são classificados, dependendo do ácido formado em sua hidrólise, em galo ou elágico taninos. Os taninos condensados são constituídos por monômeros do tipo catequina, conhecidos por flavonoides (Pizzi, 1993), e estão presente, basicamente, na casca das árvores.

Além da importância no curtimento de couros e peles, os taninos são utilizados pela indústria de petróleo como agentes dispersantes, no controle da viscosidade de argilas na perfuração de poços (Paes et al., 2006a; Tanac S.A., 2012), sendo, também, empregados no tratamento de água de abastecimento e residuárias (Pelegrino, 2011; Klumb \& Faria, 2012; Tanac S.A., 2012), na fabricação de tintas e adesivos para madeira e derivados (Trugilho et al., 1997) e, em virtude de suas propriedades antissépticas, vêm sendo testados contra organismos xilófagos (Vital et al., 2001; Trugilho et al., 2003; Colli et al., 2007).

Em decorrência do custo de aquisição de adesivos sintéticos para colagem de madeira e derivados e da importância dos taninos vegetais para a síntese de adesivo tanino formaldeído, varias pesquisas com o emprego de taninos vegetais vêm sendo desenvolvidas (Vázquez et al., 2005; Gonçalves et al., 2008; Almeida et al., 2010; Moubarik et al., 2010; Carneiro et al., 2012; Gonçalves, 2012).

No Brasil há várias espécies florestais produtoras de taninos, porém os curtumes tradicionais da Região Nordeste que utilizam taninos vegetais, apesar da diversidade de espécie arbóreas e arbustivas que ocorrem na região, têm no angico-vermelho (Anadenanthera colubrina var. cebil) sua única fonte de taninos (Paes et al., 2006a; Lima, 2011).

Pesquisas relatam novas espécies produtoras de taninos na Região Nordeste, casos da jurema-vermelha (Mimosa arenosa), do cajueiro (Anacardium occidentale) e da jurema-preta (Mimosa tenuiflora) (Paes et al., 2006c).

A jurema-preta é uma espécie pioneira encontrada com frequência no semiárido brasileiro, explorada comercialmente como fonte energética por causa do alto poder calorífico (Oliveira, 2003; Oliveira et al., 2006) e que também produz forragem e madeira para construções rurais. Paes et al. (2006b) detectaram que a casca de jurema-preta apresenta $17,74 \%$ de taninos, tendo potencial para indústrias de couros e tanantes. Azevêdo (2010) constatou que os taninos obtidos desta espécie possuem boas características para a produção de adesivo tanino formaldeído.

Este estudo teve como objetivo avaliar o teor de taninos condensados presente na casca de jurema-preta (Mimosa tenuiflora) em função das fenofases da planta, a fim de determinar a melhor época de coleta de casca para a produção de taninos.

\section{MATERIAL E MÉTODOS}

\subsection{Seleção das árvores estudadas}

A casca das árvores de jurema-preta [Mimosa tenuiflora (Willd.) Poir.] foram coletadas em uma floresta nativa, localizada na Fazenda Lameirão, de propriedade da Universidade Federal de Campina Grande, localizada no município de Santa Terezinha, Estado da Paraíba (latitude $7^{\circ} \mathrm{S}$, longitude $37^{\circ} 04^{\prime} \mathrm{W}$, altitude de 240 metros e clima, segundo a classificação de Köppen, semiárido do tipo Aw' - quente úmido, com chuvas de verão ou outono), com média anual das precipitações pluviométricas em torno de $600 \mathrm{~mm}$.

As coletas foram realizadas de acordo com o ano agrícola, com o intuito de avaliar a influência da fenologia na produção de taninos a partir da casca das árvores, tendo sido abatidas 10 árvores por coleta (agosto e dezembro de 2008 e abril e agosto de 2009), totalizando 40 árvores.

A casca das árvores foi retirada, acondicionada em sacos plásticos para evitar a perda de umidade, levada ao laboratório, pesada, posta para secar e novamente pesada, a fim de determinar o teor de umidade por período de coleta.

Para a determinação da quantidade de taninos foi tomada uma amostra representativa da quantidade de cascas obtida por coleta. Para a extração dos taninos, as cascas foram reduzidas a fragmentos menores com o uso de uma forrageira, posteriormente secas ao ar. Após a secagem, o material foi amostrado para a determinação da umidade, a fim de se calcular a 
quantidade de taninos em base seca presente em cada amostra.

Do material seco ao ar foram tomadas três amostras representativas, que foram moídas em moinho do tipo Willey para obtenção de um material de menor granulometria. Para evitar o aquecimento acentuado das facas do moinho, o que poderia causar alterações na composição química do material, o processo de moagem ocorreu com paradas constantes, sempre que o moinho aquecia.

A casca na forma de serragem foi classificada com auxílio de peneiras, sendo utilizado o material que passou pela peneira de 16 mesh $(1,00 \mathrm{~mm})$ e ficou retido na de 60 mesh $(0,25 \mathrm{~mm})$. A serragem foi homogeneizada e o teor de umidade determinado, para permitir os cálculos, em base seca, do teor de taninos presente em cada amostra.

\subsection{Extração e quantificação das substâncias tânicas}

As substâncias tânicas contidas no material foram extraídas em água destilada. Para as extrações, foram tomadas, de cada material, três amostras de $25 \mathrm{~g}$ de material seco. As amostras foram transferidas para balões de fundo chato com capacidade de $500 \mathrm{~mL}$, aos quais foram adicionados $250 \mathrm{~mL}$ de água destilada (relação 1:10), e cada amostra foi submetida a fervura sob refluxo por duas horas. Foram realizadas duas extrações de cada amostra, a fim de se retirar a máxima quantidade de extrativos presentes. Assim, a relação material:solução passou a 1:20.

Após cada extração, o material foi passado em uma peneira de 150 mesh $(0,105 \mathrm{~mm})$ e em um tecido de flanela, para a retenção de partículas finas. O extrato obtido foi homogeneizado e filtrado em funil de vidro sinterizado de porosidade 2. Em seguida foi concentrado para $250 \mathrm{~mL}$, pela evaporação da água em aparelho tipo Soxhlet, sendo retiradas três alíquotas (amostras) de $50 \mathrm{~mL}$ de cada extrato. Duas foram utilizadas para a determinação do teor de taninos condensados (TTC) e uma foi evaporada em estufa a $103{ }^{\circ} \mathrm{C} \pm 2{ }^{\circ} \mathrm{C}$ por 48 horas, para a determinação da porcentagem de teor de sólidos totais (TST) Equação 1.

$$
\operatorname{TST}(\%)=\frac{M_{i}-M_{f}}{M_{i}} \times 100
$$

em que: TST = teor de sólidos totais, em porcentagem; $M_{i}=$ massa inicial, em gramas; e $M_{f}=$ massa final, após secagem, em gramas.

Para a determinação do TTC presente em cada amostra foi empregado o método de Stiasny, descrito por Guangcheng et al. (1991), com algumas modificações. Para tanto, aos $50 \mathrm{~mL}$ do extrato bruto foram adicionados $4 \mathrm{~mL}$ de formaldeído $(37 \% \mathrm{~m} / \mathrm{m}) \mathrm{e}$ $1 \mathrm{~mL}$ de $\mathrm{HCl}$ concentrado. Cada mistura foi submetida à fervura sob refluxo por 30 minutos. Nessas condições, os taninos formam complexos insolúveis que podem ser separados por filtragem simples.

Para isso empregou-se filtro de papel posto em funil de Büchner de $10 \mathrm{~cm}$ de diâmetro e $4 \mathrm{~cm}$ de profundidade. $\mathrm{O}$ material retido no filtro foi seco em estufa a $103^{\circ} \mathrm{C} \pm 2{ }^{\circ} \mathrm{C}$ por 24 horas e o índice de Stiasny foi calculado Equação 2.

$I(\%)=\left(\frac{M_{2}}{M_{1}}\right) \times 100$

em que: I = índice de Stiasny, em porcentagem; $\mathrm{M}_{1}=$ massa de sólidos em $50 \mathrm{~mL}$ de extrato; $\mathrm{e}_{2}=$ massa do precipitado tanino-formaldeído.

A quantidade de taninos presente em cada amostra foi obtida ao se multiplicar o índice de Stiasny pelo teor de sólidos totais (Equação 3).

$\operatorname{TTC}(\%)=\frac{T S T \times I}{100}$

em que: TTC = teor de taninos condensados, em porcentagem; TST = equação 1 ; e I = equação 2 .

Todas as análises foram realizadas em triplicata. Os resultados de TST, I e TTC foram analisados estatisticamente e as médias comparadas pelo teste de Tukey a $5 \%$ de probabilidade. Os valores em porcentagem foram transformados em arcsen [raiz (dados/100)]. Essa transformação dos dados, sugerida por Steel \& Torrie (1980), foi necessária para homogeneizar as variâncias.

\section{RESULTADOS E DISCUSSÃO}

Na Caatinga, no mês de agosto, em geral ocorrem baixos índices pluviométricos, as plantas perdem as folhas (espécies caducifólias) (Figura 1), como estratégia para evitar a perda de água por transpiração (Penhalber \& Mantovani, 1997). 


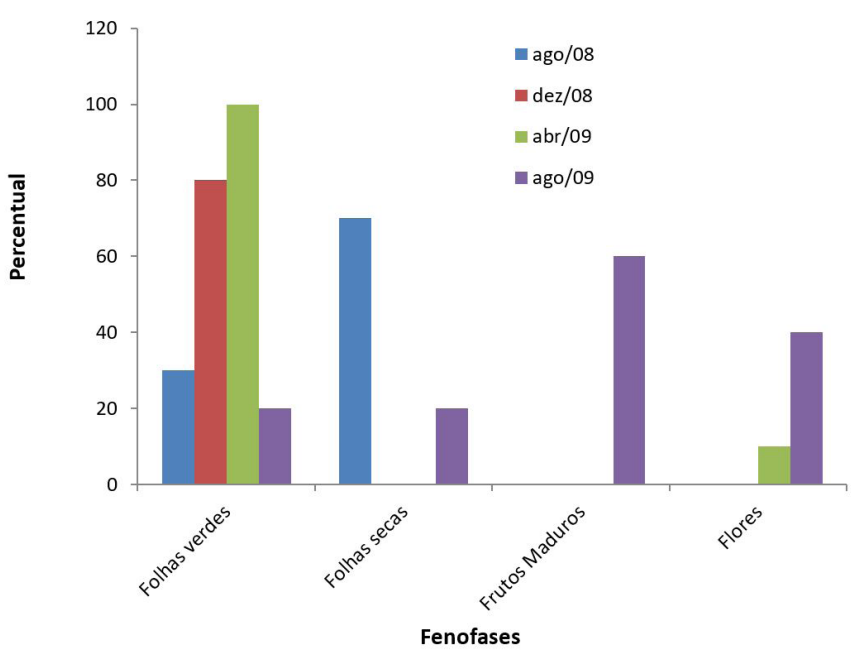

Figura 1. Percentual de eventos fenológicos (fenofases) das plantas de jurema-preta (Mimosa tenuiflora) no período de agosto de 2008 a agosto de 2009.

Figure 1. Percentage of phenological events (phenofases) at Mimosa tenuiflora plants in the period from August 2008 to August 2009.

A fenologia das espécies estudadas provavelmente foi influenciada pelo padrão de chuvas durante o período de avaliação, conforme esperado para um ambiente com estresse hídrico (Sampaio, 1995; Machado et al., 1997).

A floração ou a rebrota durante a seca, em algumas espécies, é um paradoxo (Borchert, 1994). A floração ocorreu no início do período seco (Figura 1), o mesmo foi encontrado por Figueiredo (2008). Tem sido postulado que são resposta a eventos esporádicos de precipitação durante a seca (Opler et al., 1980), ou que são restritas a espécies de regiões com seca menos severa ou savanas (Bullock, 1995). Argumenta-se ainda que espécies tolerantes, com maior capacidade de absorção e, ou, retenção de água não são limitadas pela seca, sendo ainda incertas as causas da sua periodicidade (Medina, 1995).

Conforme esperado, os padrões fenológicos observados parecem refletir o padrão sazonal das chuvas, sendo o final de período seco e o início de período chuvoso a época de frutificação encontrada nos estágios seccionais, tendo os mesmos resultados sido encontrados por Figueiredo (2008). Na Tabela 1 encontram-se os valores médios de teor de umidade em base seca para a casca de jurema-preta por período de coleta.

Os maiores teores de umidade em base seca na casca de jurema-preta foram encontrados nos meses
Tabela 1. Teor de umidade da casca de jurema-preta em base seca.

Table 1. Moisture content of Mimosa tenuiflora barks on a dry basis.

\begin{tabular}{|c|cc|}
\multicolumn{2}{|c|}{ Período de coleta } & Teor de umidade (\%) \\
\hline \multirow{2}{*}{2008} & Agosto & 47,62 \\
& Dezembro & 90,60 \\
\multirow{2}{*}{2009} & Abril & 83,50 \\
\cline { 2 - 3 } & Agosto & 67,22 \\
\hline
\end{tabular}

de dezembro de 2008 e abril de 2009, os quais, segundo a fenologia, foram os meses que apresentaram maior percentual de folhas na copa, enquanto o menor teor de umidade foi encontrado no mês de agosto de 2008. Na Tabela 2 é possível encontrar a concentração de taninos, teor de sólidos totais e índice de Stiasny presente na casca de jurema-preta.

$\mathrm{Na}$ avaliação de todas as análises, o efeito dos fatores foi dependente $(\mathrm{P}<0,05)$, havendo diferença significativa entre os meses de coleta de acordo com a fenologia. O teor de taninos condensados (TTC) foi maior no mês de dezembro de 2008, em que a planta se encontrava apenas com presença de folhas verdes (80\%).

O menor teor de taninos condensados foi encontrado no mês de agosto de 2009, valor próximo ao obtido por Paes et al. (2006b), que foi de 17,74\% para a mesma espécie. Isso deve ter ocorrido pela 
Tabela 2. Valores médios do teor de sólidos totais (TST), índice de Stiasny (I) e teor de taninos condensados (TTC) encontrados na casca de jurema-preta.

Table 2. Average values of total solids content (TST), Stiasny's index (I) and condensed tannins content (TTC) found in Mimosa tenuiflora barks.

\begin{tabular}{|c|c|c|c|c|}
\hline \multicolumn{2}{|c|}{ Período de coleta } & $\begin{array}{l}\text { TST } \\
(\%)\end{array}$ & $\begin{array}{c}\text { I } \\
(\%)\end{array}$ & $\begin{array}{c}\text { TTC } \\
(\%)\end{array}$ \\
\hline \multirow{2}{*}{2008} & Agosto & $25,20 \mathrm{~b}$ & $69,84 \mathrm{a}$ & $17,60 \mathrm{~b}$ \\
\hline & Dezembro & $30,80 \mathrm{a}$ & $71,12 \mathrm{a}$ & $21,90 \mathrm{a}$ \\
\hline \multirow{2}{*}{2009} & Abril & $29,40 \mathrm{a}$ & $67,35 \mathrm{ab}$ & $19,80 \mathrm{ab}$ \\
\hline & Agosto & $24,25 \mathrm{~b}$ & $65,70 \mathrm{~b}$ & $16,04 \mathrm{~b}$ \\
\hline
\end{tabular}

Médias seguidas por letras minúsculas iguais na mesma coluna não diferem entre si (Tukey, $p>0,05$ ).

grande presença de frutos (60\%) nesse mês (Figura 1). Paes et al. (2010) observaram que o angico-vermelho (Anadenanthera colubrina) apresentou um teor de $10,69 \%$ de taninos nos frutos. Por essa razão, acredita-se que a planta se utiliza de uma estratégia em que os taninos são direcionados da casca para os frutos, evitando assim o ataque de predadores, por causa de suas propriedades antissépticas contra insetos xilófagos (Couto, 1996; Vital et al., 2001), reduzindo-se assim o teor de taninos condensados na casca.

Para o índice de Stiasny, não houve diferença significativa nos meses de agosto e dezembro de 2008 e abril de 2009. Agosto de 2009 apresentou o menor índice, porém não diferindo do de abril de 2009, que apresentou um valor intermediário entre os meses avaliados. Esse índice indica que em agosto e dezembro de 2008, no extrato de plantas com maior presença de folhas verdes ou secando (Figura 1) existia uma maior quantidade de substâncias fenólicas, as quais reagiram com o formaldeído em meio ácido (Guangcheng et al., 1991; Mori, 1997; Carneiro et al., 2001), em relação ao das plantas colhidas no mês de agosto de 2009, que estavam com frutos secos e botões florais. Paes et al. (2006b) observaram que no período em que as árvores de angico-vermelho (Anadenanthera colubrina) estavam com folhas verdes houve uma maior quantidade de taninos nas cascas.

Já para o teor de sólidos totais (TST) não houve diferença significativa entre dezembro de 2008 e abril de 2009, com os maiores valores. Os meses de agosto de 2008 e 2009 apresentaram os menores valores. Os valores médios de TST para os quatro períodos avaliados, igual a $26,16 \%$, foi semelhante aos obtidos por Paes et al. (2006a) e Lima (2011), que foi de 26,32\% para a mesma espécie avaliada.

\section{CONCLUSÕES}

O teor de taninos presente na casca de jurema-preta foi afetado pelas fenofases da planta.

Os menores valores para teor de taninos, teor de sólidos totais e índice de Stiasny presentes na casca de jurema-preta foram obtidos para o período de frutificação das árvores.

O maior teor de taninos da casca de jurema-preta foi obtido quando as plantas estavam com maior presença de folhas verdes (dezembro de 2008 e abril de 2009).

Seria interessante realizar estudos para comprovar o teor de taninos presente nos frutos e raízes de jurema-preta.

\section{STATUS DA SUBMISSÃO}

Recebido: 6 jan., 2013

Aceito: 26 jun., 2016

\section{AUTOR(ES) PARA CORRESPONDÊNCIA}

\section{Juarez Benigno Paes}

Departamento de Ciências Florestais e da Madeira, Universidade Federal do Espírito Santo - UFES, Av. Governador Lindemberg, 316, Centro, CEP 29550-000, Jerônimo Monteiro, ES, Brasil

e-mail: jbp2@uol.com.br

\section{REFERÊNCIAS}

Almeida NF, Mori FA, Goulart SL, Mendes LM. Estudo da reatividade de taninos de folhas e cascas de barbatimão Stryphnodendron adstringens (Mart.) Coville. Scientia Forestalis 2010; 38(87): 401-408. 
Azevêdo TKB. Relações entre madeira, casca e teor de substâncias tânicas e qualidade dos taninos de jurema-preta (Mimosa tenuiflora (Willd.) Poir.) para produção de adesivos tanino formaldeído [dissertação]. Patos: Universidade Federal de Campina Grande; 2010. 76 p.

Borchert R. Soil and stem water storage determine phenology and distribution of tropical dry forest trees. Ecology 1994; 75(5): 1437-1449. http://dx.doi.org/10.2307/1937467.

Bullock SH. Plant reproduction in neotropical dry forest trees. In: Bullock SH, Mooney HA, Medina E, editors. Seasonally dry tropical forests. Cambridge: Cambridge University Press; 1995. p. 277-296.

Carneiro ACO, Vital BR, Castro AFNM, Santos RC, Castro RVO, Pinheiro MA. Parâmetros cinéticos de adesivos produzidos a partir de taninos de Anadenanthera peregrina e Eucalyptus grandis. Revista Árvore 2012; 36(4): 767-775. http://dx.doi.org/10.1590/S0100-67622012000400018.

Carneiro ACO, Vital BR, Pimenta AS, Mori FA. Reatividade dos taninos da casca de Eucalyptus grandis para produção de adesivos. Cerne 2001; 7(1): 1-9.

Colli A, Nascimento AM, Xavier LM, Rubim IB. Propriedades físico-mecânicas e preservação, com boro e tanino, do Bambusa tuldoides (Munro). Floresta e Ambiente 2007; 14(1): 56-64.

Couto LC. Potentiel fongicide dês extraits d'écorce de barbatimão à létat brut et combines aux íons $\mathrm{Fe}+++$ et Al+++ [tese]. Laval: Faculté de Foresterie et de Géomatique: Université Laval; 1996. 262 p.

Figueiredo PS. Fenologia e estratégias reprodutivas das espécies arbóreas em uma área marginal de cerrado, na transição para o semi-árido no nordeste do Maranhão, Brasil. Revista Trópica: Ciências Agrárias e Biológicas 2008; 2 (2): 8-22.

Gonçalves FG, Lelis RCC, Oliveira JTS. Influência da composição da resina tanino-uréia-formaldeído nas propriedades físicas e mecânicas de chapas aglomeradas. Revista Árvore 2008; 32(4): 715-722. http://dx.doi. org/10.1590/S0100-67622008000400013.

Gonçalves FG. Painéis aglomerados de madeira de Acacia mangium com adesivos de uréia formaldeído e tanino em pó da casca de Acacia mearnsii [tese]. Seropédica: Universidade Federal Rural do Rio de Janeiro; 2012.

Guangcheng Z, Yunlu L, Yazaki Y. Extractive yields, Stiasny values and polyflavonoid contents in barks form six acacia species in Australia. Australian Forestry 1991; 554(2): 154-156. http://dx.doi.org/10.1080/00049158.1 991.10674572.

Klumb AK, Faria OLV. Produção de coagulante vegetal catiônico a partir de cascas de eucalipto (Eucalyptus tereticornis). Vetor 2012; 22(1): 71-80.

Lima CR. Potencialidade dos extratos tânicos das espécies angico vermelho, jurema preta e jurema-vermelha no curtimento de peles caprinas [tese]. Campina Grande: Universidade Federal de Campina Grande; 2011. 61 p.

Machado IC, Barros LM, Sampaio EVSB. Phenology of Caatinga species at Serra Talhada, PE, Northeastern Brazil. Biotropica 1997; 29(1): 57-68. http://dx.doi. org/10.1111/j.1744-7429.1997.tb00006.x.

Medina E. Diversity of life forms of higher plants in neotropical dry forests. In: Bullock SH, Mooney HA, Medina E, editors. Seasonality dry tropical forests. Cambridge: Cambridge University Press; 1995. p. 221-238.

Mori FA. Uso de taninos da casca de Eucalyptus grandis para produção de adesivos [dissertação]. Viçosa: Universidade Federal de Viçosa; 1997. 47 p.

Moubarik A, Allal A, Pizzi A, Charrier F, Charrier B. Preparation and mechanical characterization of particleboard made from maritime pine and glued with bio-adhesives based on cornstarch and tannins. Maderas. Ciencia $y$ Tecnología 2010; 12(3): 189-197.

Oliveira E, Vital BR, Pimenta AS, Della Lucia RM, Ladeira AMM, Carneiro ACO. Estrutura anatômica da madeira e qualidade do carvão de Mimosa tenuiflora (Willd.) Poir. Revista Árvore 2006; 30(2): 311-318. http://dx.doi. org/10.1590/S0100-67622006000200018.

Oliveira E. Características anatômicas, químicas e térmicas da madeira de três espécies de maior ocorrência no SemiÁrido Nordestino [tese]. Viçosa: Universidade Federal de Viçosa; 2003. 122 p.

Opler AP, Frankie GW, Baker HG. Comparative phenological studies of treelet and shrub species in a tropical wet and dry forest in the lowlands of Costa Rica. Journal of Ecology 1980; 68(1): 167-188. http://dx.doi.org/10.2307/2259250.

Paes JB, Diniz CEF, Marinho IV, Lima CR. Avaliação do potencial tanífero de seis espécies florestais de ocorrência no semi-árido brasileiro. Cerne 2006a; 12(3): 232-238.

Paes JB, Lima CR, Oliveira E, Azevedo TKB. Variação no teor de taninos com as fenofases da planta e posições no tronco em árvores de angico vermelho (Anadenanthera colubrina (Vell.) Brenan var. cebil (Gris.) Alts.). In: Anais do X Encontro Brasileiro em Madeiras e em Estruturas de Madeira [CD-ROM]; 2006; São Pedro. São Pedro: CEVEMAD: UNESP: IBRAMEM; 2006b.

Paes JB, Marinho IV, Lima RA, Lima CR, Azevedo TKB. Viabilidade técnica dos taninos de quatro espécies florestais de ocorrência no semi-árido brasileiro no curtimento de peles. Ciência Florestal 2006c; 16(4): 453-462. http:// dx.doi.org/10.5902/198050981927.

Paes JB, Santana GM, Azevedo TKB, Morais RM, Calixto Júnior JT. Substâncias tânicas presentes em várias partes da árvore angico-vermelho (Anadenanthera colubrina (Vell.) Brenan. var. cebil (Gris.) Alts.). Scientia Forestalis 2010; 38(87): 441-447.

Pelegrino ECF. Emprego de coagulante à base de tanino em sistema de pós-tratamento de efluente de reator UASB por 
flotação [dissertação]. São Carlos: Universidade de São Paulo, Escola de Engenharia de São Carlos; 2011. 155 p.

Penhalber EF, Mantovani W. Floração e chuva de sementes em mata secundária em São Paulo, SP. Revista Brasileira de Botanica. Brazilian Journal of Botany 1997; 20(2): 205-220. http://dx.doi.org/10.1590/S0100-84041997000200011.

Pizzi A. Tanin-based adhesives. In: Pizzi A, editor. Wood adhesives: chemistry and technology. New York: Marcell Dekker; 1993. p. 177-246.

Sampaio EVSB. Overview of the Brazilian Caatinga. In: Bullock SH, Mooney HA, Medina E, editors. Seasonally dry tropical forests. Cambridge: Cambridge University Press; 1995. p. 34-63.

Steel RGD, Torrie JH. Principles and procedures of statistic: a biometrical approach. 2nd ed. New York: Mc-Graw Hill; 1980. 633 p.

Tanac S.A. Construindo o futuro todos os dias [online]. [citado em 2012 dez. 15]. 2012. Disponível em: http://
www.tanac.com.br/PT/institucional.php?codCategoria $\mathrm{Menu}=148 \&$ nomArea $=$ Hist $\% \mathrm{C} 3 \% \mathrm{~B} 3 \mathrm{rico} \& \operatorname{codDado}=$ $2 \&$ menu $=138$

Trugilho PF, Caixeta RP, Lima JT, Mendes LM. Avaliação do conteúdo em taninos condensados de algumas espécies típicas do cerrado mineiro. Cerne 1997; 3(1): 1-13.

Trugilho PF, Mori FA, Lima JT, Cardoso DP. Determinação do teor de taninos na casca de Eucalyptus spp. Cerne 2003; 9(2): 246-254

Vázquez G, López-Suevos F, González-Álvarez J, Antorrena G. Adhesivos fenol-urea-formaldehído modificados con taninos para contrachapados de uso exterior. Información Tecnológica 2005; 16(2): 41-46.

Vital BR, Shimada AN, Della Lucia RM, Valente OF, Pimenta AS. Avaliação dos taninos da casca de Eucalyptus grandis W. Hill ex Maiden como preservativo de madeira. Revista Árvore 2001; 25(2): 245-256. 